Case Report

\title{
Cutaneous Vasculitis, Interstitial Pneumonia with Crazy-Paving Appearance, and Positive pANCA in a Patient with Severe Crohn's Disease
}

\author{
Guang-liang Chen, ${ }^{1,2}$ Juan Wang, ${ }^{3}$ Bao-zhen Li, ${ }^{1}$ Li-mei Li, ${ }^{1}$ Han-you Mo, ${ }^{1}$ and Shuang Ye \\ ${ }^{1}$ Department of Rheumatology \& Immunology, Affiliated Hospital of Guilin Medical University, 5th Floor, Building No. 3, \\ 15 Lequn Road, Guilin 541001, China \\ ${ }^{2}$ Department of Internal Medicine 3, University of Erlangen-Nuremberg, 91054 Erlangen, Germany \\ ${ }^{3}$ Department of Rheumatology, Huashan Hospital Affiliated to Fudan University, Shanghai 200040, China \\ ${ }^{4}$ Department of Rheumatology, Renji Hospital Affiliated to Shanghai Jiao Tong University School of Medicine, \\ 145 Shandong C Road, Shanghai 200001, China
}

Correspondence should be addressed to Han-you Mo; wjcglyiqi@163.com and Shuang Ye; yeshuang@online.sh.cn

Received 30 March 2014; Revised 12 July 2014; Accepted 5 August 2014; Published 14 August 2014

Academic Editor: Ian Craig Lawrance

Copyright (C) 2014 Guang-liang Chen et al. This is an open access article distributed under the Creative Commons Attribution License, which permits unrestricted use, distribution, and reproduction in any medium, provided the original work is properly cited.

Cutaneous vasculitis, interstitial pneumonia with crazy-paving appearance on high-resolution computed tomography, and repeated positive perinuclear anti-neutrophil cytoplasmic antibodies (pANCA) are rarely found together in patients with inflammatory bowel disease in the existing literature. We report the case of a Chinese patient previously diagnosed with cutaneous vasculitis and interstitial pneumonia, who presented with acute pain and mass in his right lower quadrant a couple of years later. The terminal ileum biopsy and postoperative pathology confirmed Crohn's disease (CD).

\section{Introduction}

Extraintestinal manifestations of Crohn's disease (CD) are prevalent [1-5]. However, the diversity and nonspecific manifestations can be a challenge to physicians in managing this disease. Usually, these manifestations occur in the late course of CD and involve nearly any organ system, including the dermatologic and pulmonary systems [2-7]. It is seldom reported that extraintestinal manifestation is the first symptom of CD. We, herein, report a Chinese patient with $\mathrm{CD}$, whose cutaneous vasculitis concomitant with pulmonary manifestation antedates the onset of CD by approximately 3 years. Interestingly, the pulmonary manifestation presented as interstitial pneumonia with a crazy-paving appearance (CPA) on high-resolution computed tomography (HRCT) in the lungs.

\section{Case Presentation}

A 55-year-old Chinese male developed recurrent skin eruptions, which were tender and pruritus and lasted approximately 1 year. Skin lesions (Figure 1(a)) appeared around the entire body, especially on the lower extremities, which presented as ulcerations and healed slowly with pigmentation. He had nonproductive cough and severe dyspnea on exertion and was initially admitted to a local primary hospital in May 2010. The chest X-ray showed patchy and diffuse interstitial infiltration in both lungs (Figure 2(a)). The patient was transferred to our department in June 2010.

The medical history included chronic varicosity on the left lower extremity with 30 years of duration and enlargement of axillar and inguinal lymph nodes for 2 years. He was a nonsmoker and had a job in poultry cultivation since he was young. Elevated erythrocyte sedimentation rate (ESR, $0-15 \mathrm{~mm} / \mathrm{h}$ ) of $60 \mathrm{~mm}$ in the first hour, c-reactive protein $(\mathrm{CRP}, \leq 1 \mathrm{mg} / \mathrm{dL})$ of $10.17 \mathrm{mg} / \mathrm{dL}$, serum $\operatorname{lgG}(8-16 \mathrm{~g} / \mathrm{L})$ of $39.3 \mathrm{~g} / \mathrm{L}$, serum globulin $(25-30 \mathrm{~g} / \mathrm{L})$ of $42.82 \mathrm{~g} / \mathrm{L}$, and thyroid-stimulating hormone (TSH, $0.7-6.4 \mu \mathrm{IU} / \mathrm{mL}$ ) of $6.4 \mathrm{IU} / \mathrm{mL}$ were appreciated, while the blood cell count, urine analysis, and the rest of the biochemical studies were normal. Assays including human immunodeficiency virus 


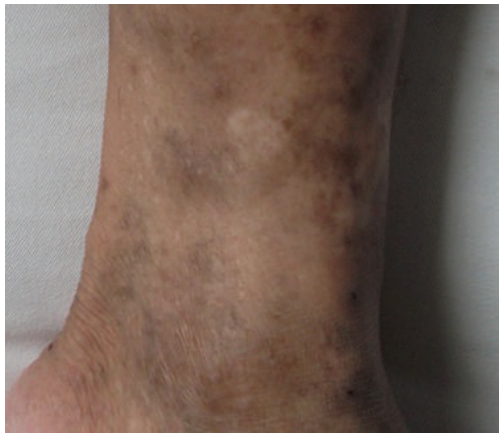

(a)

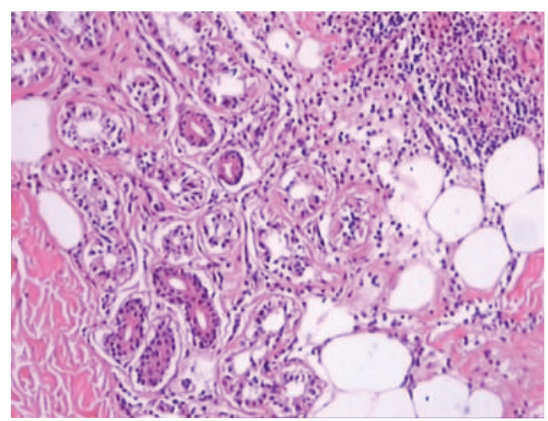

(d)

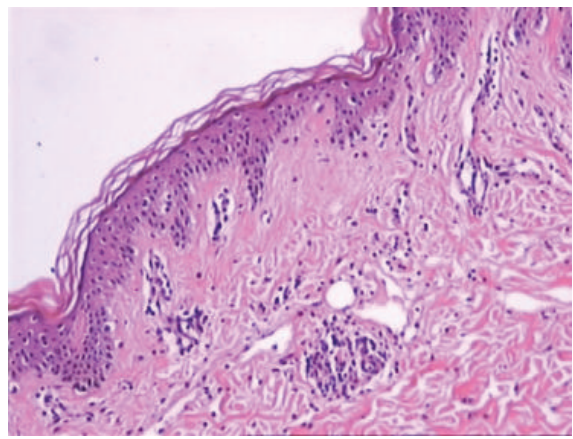

(b)

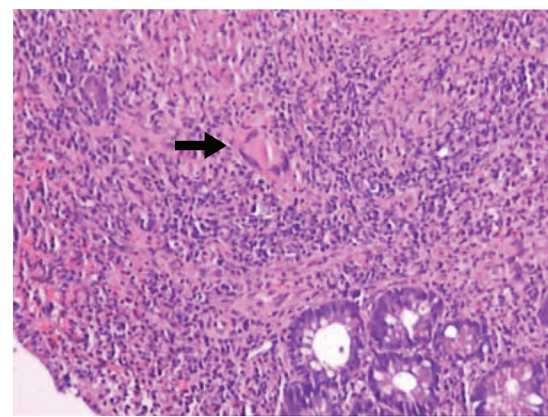

(e)

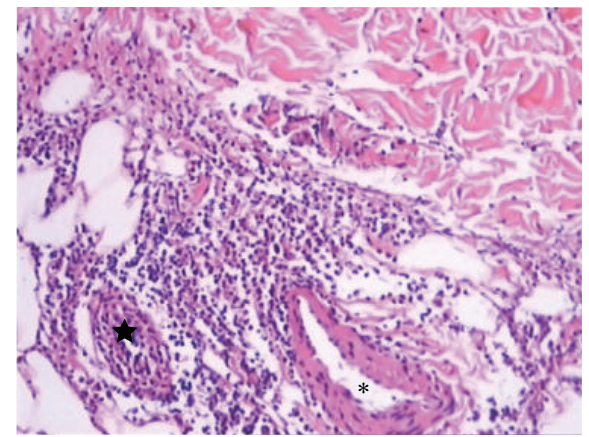

(c)

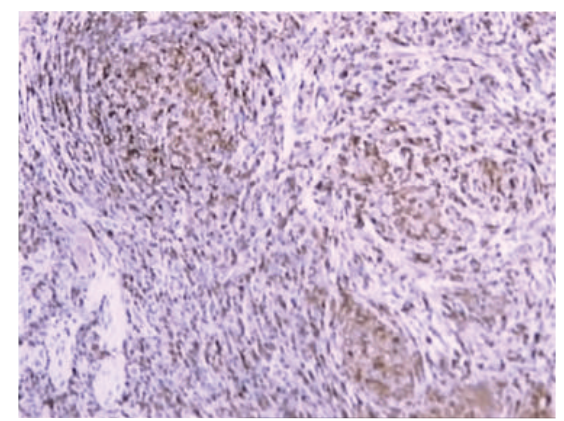

(f)

FIGURE 1: Skin lesions and pathologic findings. (a) A representative picture for skin nodules. ((b)-(d)) Histological examination of skin lesions showed mild atrophy of the epidermis and enormous neutrophilic and lymphocytic infiltration of adipose tissue and appendages in the dermal layer ((b), (d)); local small abscesses were formed (star) (c); in line with vasculitis, a thickening of the arterial walls of some small arteries by an inflammatory lymphoid infiltrate (snowflake) (H.E. stain $\times 100)$ (c). ((e)-(f)) Biopsy from the terminal ileum mucosa showed a granulomatous inflammatory infiltration (black bold arrow) (H.E. stain $\times 100)(\mathrm{e})$. Antiacid staining and PAM were negative. CD68 antigen in terminal ileum mucosa was positive $(\times 100)(\mathrm{f})$.

(HIV), hepatitis A virus (HAV), hepatitis B virus (HBV), hepatitis $\mathrm{C}$ virus (HCV), syphilis serology (RPR), purified protein derivative (PPD), and tumor markers were negative. Serodiagnostics for Clonorchis sinensis and Cysticercus cellulosae were also negative. Anti-cardiolipin antibodies (ACL-lgG/lgM) and pANCA (determined by enzyme-linked immunosorbent assay (ELISA)) were positive. Anti-nuclear antibody (ANA) was a borderline positive in a titre of $1: 100$, while ENA and complement were normal. HRCT of the chest showed diffuse ground-glass opacities and a crazypaving appearance (CPA) (Figures 2(c)-2(f)). Abdominal ultrasound showed mild enlargement in the spleen $(47 \mathrm{~mm}$ thickness). Electrocardiogram was unremarkable. Biopsy from one of the skin lesions and enlarged inguinal lymph nodes revealed nonspecific vasculitis (Figures $1(\mathrm{~b})-1(\mathrm{~d})$ ) and lymphadenitis, respectively.

He was diagnosed with cutaneous vasculitis and interstitial pneumonia. He was treated with corticosteroid (40 mg i.v. methylprednisolone) and cyclophosphamide (CYC; $0.6 \mathrm{~g} \sim 1.2 \mathrm{~g}$ was administered intravenously once a month for 6 months and subsequently once every 3 months). Thalidomide (50 mg/day) was also added orally. Skin eruptions resolved and the symptom of dyspnea improved in parallel with radiographic changes (Figure 2(d)). In September 2010, after a herpes zoster infection, the steroid dose was reduced to $7.5 \mathrm{mg} /$ day. Since the cumulative dose had reached
$12 \mathrm{~g}$ in December 2011, CYC was discontinued and hydroxychloroquine (HCQ) (400 mg/day) was initiated. However, no further improvement of the interstitial lung disease could be appreciated (Figures 2(e) and 2(f)).

On January 3rd, 2012, the patient was admitted again for evaluation of two-week acute-onset abdominal pain. Physical examination revealed that the patient was hemodynamically stable and had middle-grade fever. A mild tenderness on the right lower quadrant was found. There was no progression in skin eruptions on the lower extremities. A HRCT scan of the abdomen showed severe segmental narrowing and a cobblestone appearance in the terminal ileum (Figure 3). Ulcerations in the mucous were revealed by colonoscopy. Biopsy from the mucous verified a granulomatous inflammatory infiltration (Figures 1(e) and 1(f)). The patient was empirically treated with a standard anti-TB therapy for nearly 3 months without improvement. A repeated colonoscopy was performed. The transverse colon mucosa near the hepatic flexure was swollen and had a nodular appearance. The pathologic finding showed severe inflammation and mucosal ulceration with a large number of lymphocytes and neutrophil infiltration but without granulomas. In the middle of April 2012, a third colonoscopy showed severe acute and chronic inflammation in the terminal ileum mucosa accompanied by chronic ulcerations (some areas presented as fissurelike lesions). Moreover, the acid-fast staining and PCR for 


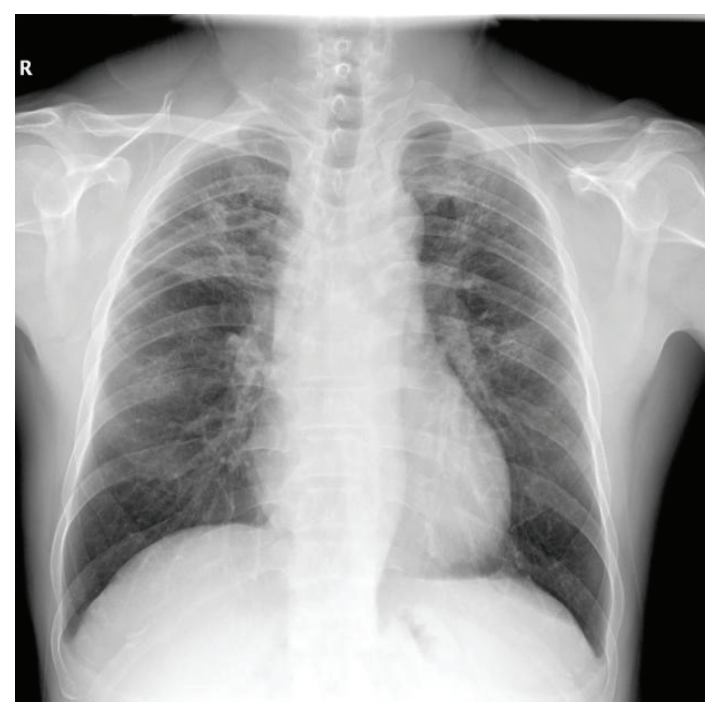

(a)

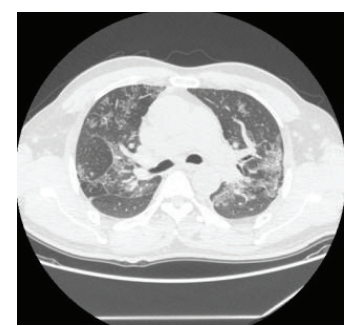

(c)

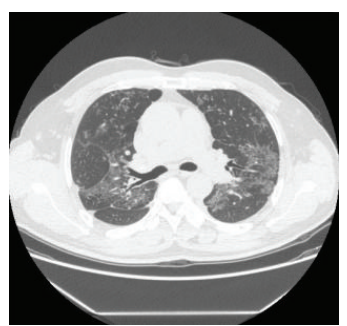

(d)

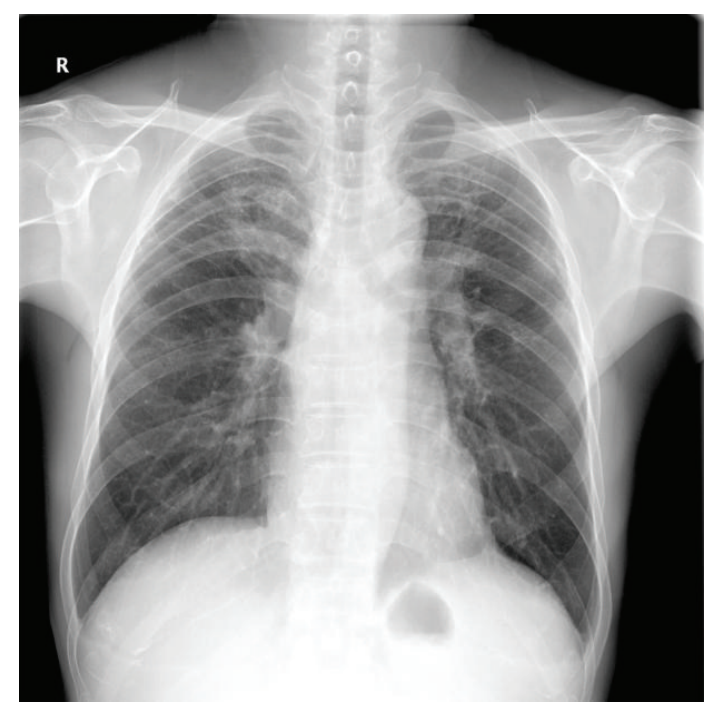

(b)

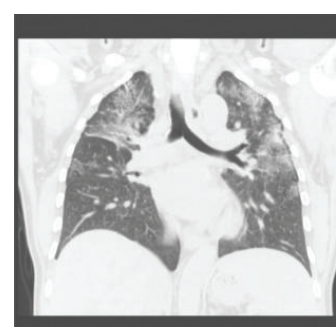

(f)

Figure 2: X-ray films of the chest were taken in June 2010 (a) and September 2012 (b). High-resolution computed tomography (HRCT) showed diffuse ground-glass opacities and a crazy-paving appearance in both lungs. (c), (d), and (e) were taken in July 2010, December 2010, and June 2011, respectively. (f), which was taken in June 2011, showed the lesions in both of the upper lungs.

Mycobacterium tuberculosis were all negative. Anti-TB treatment was stopped, and the patient was switched to methylprednisolone (MP) plus mesalazine. But he deteriorated with an abdominal intestinal fistula formation and lost nearly $20 \mathrm{~kg}$ weight in 3 months. A subtotal colectomy and ileostomy were performed in June 2012. The postoperative pathologic findings of the intestines showed pathologic changes typical of $\mathrm{CD}$. The lymph nodes showed reactive mesangial hyperplasia and hyaline degeneration. Acid-fast staining, periodic acid-Schiff (PAS), and periodic acid methenamine (PAM) in the mucosa were negative. After the surgical treatment, he recovered gradually. The reexamination of the chest Xray was also improved significantly (Figure 2(b)), while the pANCA testing was still strongly positive. The patient received corticosteroid $(5 \mathrm{mg} /$ day) and tripterygium glycosides (30 mg/day) as maintenance and remained stable in the subsequent 2-year follow-up.

\section{Discussion}

The coexistence of cutaneous vasculitis and interstitial lung disease in CD has never been reported. To the best of our knowledge, this is the first case indicating that the pulmonary manifestation in CD could present as interstitial pneumonia with a CPA on HRCT in the lungs.
Skin lesions occur in 14-44\% of patients with CD [1]. However, as an initial symptom of CD, cutaneous vasculitis was seldom found in the published literature. Mader et al. [2] searched in the MEDLINE database and only one case presented with cutaneous leukocytoclastic vasculitis as the initial manifestation of CD 3 months before the bowel disease was reported. Similarly, Tsiamoulos et al. [3] reported a case of an octogenarian with cutaneous vasculitis in the skin as the first symptom of CD 2 months before the bowel disease. The typical pathology patterns of these CDrelated cutaneous lesions include vasculitis, extravascular neutrophilia, and granulomatous inflammation [1]. In the present case, cutaneous vasculitis occurred 3 years prior to the bowel disease with relatively nonspecific vasculitic changes. However, the link between the cutaneous lesions and $\mathrm{CD}$ in this patient cannot be excluded. Consistent with the observations reported by Tsiamoulos et al. [3] and Zlatanic et al. [4], the skin lesions related to CD likely have a good response to the immunosuppressant therapy. And, in our case, the aggressive immunosuppressive treatment for cutaneous vasculitis and interstitial lung disease may in fact retard the subsequent development of full-blown CD.

Pulmonary involvement in $\mathrm{CD}$ has previously been reported with an estimated prevalence of $0.4 \%$ in a study of 1400 patients [5-7]. The airways and lung parenchyma were 


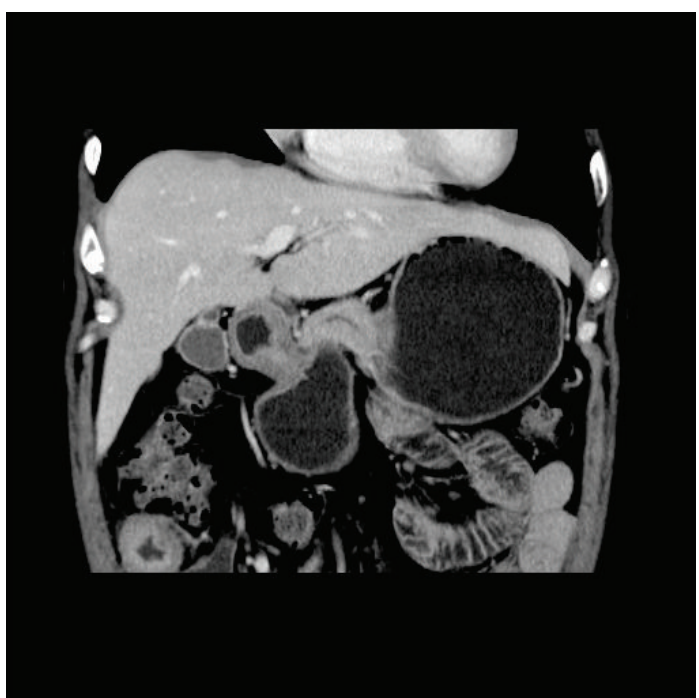

(a)

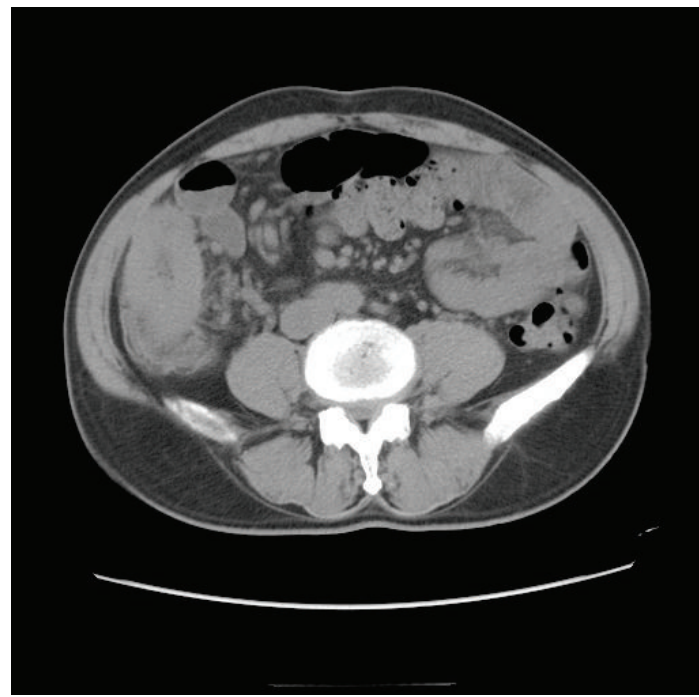

(c)

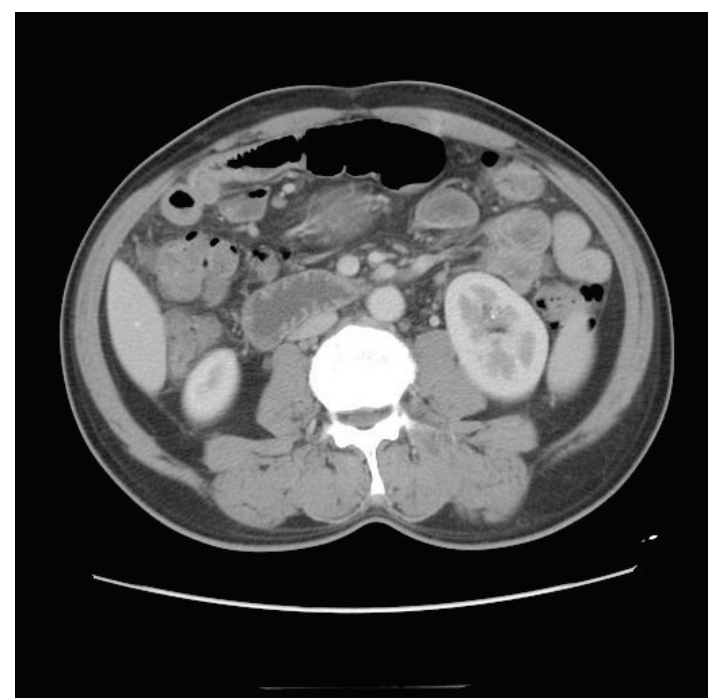

(b)

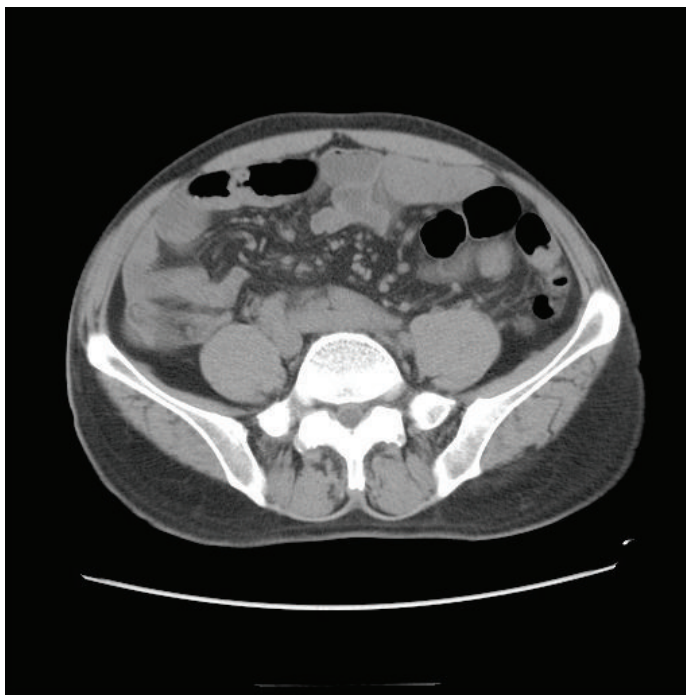

(d)

FIGURE 3: HRCT scan of the abdomen showed severe segmental narrowing, a cobblestone appearance of the terminal ileum, small-bowel wall thickening, and mesenteric adenopathy.

more commonly affected by granulomatous inflammation or nongranulomatous inflammation owing to the preexisting CD [5-7]. Shulimzon et al. [8] described two female patients with $\mathrm{CD}$ whose necrotizing granulomatous inflammation in the lung occurred 5 years before the bowel symptoms. Roblin et al. [9] also reported a pediatric case with granulomatous pulmonary involvement before the diagnosis of $\mathrm{CD}$. In our case, pulmonary manifestation antedates the onset of CD by nearly 2 years with a CPA pattern found by HRCT. CPA has a variety of causes, including infectious, neoplastic, idiopathic, inhalational, and autoimmune disorders [10]. However, CPA has never been reported in CD patients. Despite the lack of open lung biopsy data, the observation of the symptoms and radiographic findings of the lungs subsided after the surgery suggested this connection. Indeed, it has been reported [11] in a case series that included seven CD patients where the disease activity in the pulmonary and intestine paralleled.
CD is characterized as a granulomatous systemic autoimmune disease, and 10\% 20\% of the patients presented with a variety of autoantibodies [12-14]. In 1996, Vasiliauskas et al. suggested that pANCA identifies a subset of CD with a UC(ulcerative-colitis-) like presentation [13], but this cannot be validated in an independent cohort [14]. Here, the pANCA testing of the patient was repeatedly positive without a UClike presentation, suggesting the presence of genetic heterogeneity in pANCA as a biomarker for the disease, which was also involved in the data shown by Prideaux et al. in 2013 [15]. However, serological markers seem to be unable to indicate disease activity according to the study by Rieder et al. [16]. Likewise, we showed that pANCA is stable during the course of the disease, indicating that pANCA is another such serological marker. In addition, there are several reports in patients with coexisting rheumatologic disorder and CD, such as Wegener's granulomatosis [17], SLE [18], 
and Takayasu's arteritis [19]. Nevertheless, the presence of pANCA in our case may explain the vasculitic components of the cutaneous lesion and interstitial lung disease, albeit a specific diagnosis of ANCA-associated vasculitis cannot be established.

\section{Abbreviations}

ANCA: Anti-neutrophil cytoplasmic antibodies

ANA: Anti-nuclear antibody

ACL: Anti-cardiolipin antibodies

CRP: C-reactive protein

CD: Crohn's disease

CYC: Cyclophosphamide

ESR: Erythrocyte sedimentation rate

ELISA: Enzyme-linked immunosorbent assay

HIV: Human immunodeficiency virus

HAV: Hepatitis A virus

HBV: Hepatitis B virus

HCV: Hepatitis $\mathrm{C}$ virus

HRCT: High-resolution computed tomography

HCQ: Hydroxychloroquine

MP: $\quad$ Methylprednisolone

Lg: Immunoglobulin

RF: $\quad$ Rheumatoid factor

RPR: Rapid plasma reagin

PPD: $\quad$ Purified protein derivative

PAS: $\quad$ Periodic acid-Schiff

PAM: Periodic acid methenamine

PCR: Polymerase chain reaction

pANCA: Perinuclear anti-neutrophil cytoplasmic antibodies

TSH: Thyroid-stimulating hormone

UC: $\quad$ Ulcerative colitis.

\section{Consent}

Informed consent from the patient was obtained for this case report.

\section{Conflict of Interests}

The authors declare that there is no conflict of interests regarding the publication of this paper.

\section{Acknowledgments}

The authors thank pathologist Juan Tang and Liming Lin for providing the pathology findings and valuable suggestions. They appreciate Dr. Yubin Luo and Dr. Mireia Garcia for revising the paper. This study was supported by Grants from the Chinese National Natural Science Foundation (nos. 81160376 and 81360462).

\section{References}

[1] A. N. Crowson, G. J. Nuovo, M. C. Mihm Jr., and C. Magro, "Cutaneous manifestations of Crohn's disease, its spectrum, and its pathogenesis: intracellular consensus bacterial 16S rRNA is associated with the gastrointestinal but not the cutaneous manifestations of Crohn's disease," Human Pathology, vol. 34, no. 11, pp. 1185-1192, 2003.

[2] R. Mader, O. Segol, M. Adawi, P. Trougoboff, and E. Nussinson, "Arthritis or vasculitis as presenting symptoms of Crohn's disease," Rheumatology International, vol. 25, no. 6, pp. 401-405, 2005.

[3] Z. Tsiamoulos, G. Karamanolis, D. Polymeros, K. Triantafyllou, and T. Oikonomopoulos, "Leukocytoclastic vasculitis as an onset symptom of Crohn's disease," Case Reports in Gastroenterology, vol. 2, no. 3, pp. 410-414, 2008.

[4] J. Zlatanic, M. Fleisher, M. Sasson, P. Kim, and B. I. Korelitz, "Crohn's disease and acute leukocytoclastic vasculitis of skin," The American Journal of Gastroenterology, vol. 91, no. 11, pp. 2410-2413, 1996.

[5] N. D’Andrea, R. Vigliarolo, and C. M. Sanguinetti, "Respiratory involvement in inflammatory bowel diseases," Multidisciplinary Respiratory Medicine, vol. 5, no. 3, pp. 173-182, 2010.

[6] H. Omori, H. Asahi, Y. Inoue, T. Irinoda, and K. Saito, "Pulmonary involvement in Crohn's disease: report of a case and review of the literature," Inflammatory Bowel Diseases, vol. 10, no. 2, pp. 129-134, 2004.

[7] G. Hotermans, A. Benard, H. Guenanen, G. Demarcq-Delerue, T. Malart, and B. Wallaert, "Nongranulomatous interstitial lung disease in Crohn's disease," The European Respiratory Journal, vol. 9, no. 2, pp. 380-382, 1996.

[8] T. Shulimzon, J. Rozenman, M. Perelman, E. Bardan, and I. BenDov, "Necrotizing granulomata in the lung preceding colonic involvement in 2 patients with Crohn's disease," Respiration, vol. 74, no. 6, pp. 698-702, 2007.

[9] E. Roblin, N. Pecciarini, H. Yantren et al., "Granulomatous pulmonary involvement preceding diagnosis of Crohn disease: a pediatric case report," Archives de Pediatrie, vol. 17, no. 9, pp. 1308-1312, 2010.

[10] S. E. Rossi, J. J. Erasmus, M. Volpacchio, T. Franquet, T. Castiglioni, and H. Page McAdams, "Crazy-paving' pattern at thin-section CT of the lungs: radiologic-pathologic overview," Radiographics, vol. 23, no. 6, pp. 1509-1519, 2003.

[11] B. Basseri, P. Enayati, A. Marchevsky, and K. A. Papadakis, "Pulmonary manifestations of inflammatory bowel disease: case presentations and review," Journal of Crohn's and Colitis, vol. 4, no. 4, pp. 390-397, 2010.

[12] C. Roozendaal and C. G. Kallenberg, "Are anti-neutrophil cytoplasmic antibodies (ANCA) clinically useful in inflammatory bowel disease (IBD)?” Clinical \& Experimental Immunology, vol. 116, no. 2, pp. 206-213, 1999.

[13] E. A. Vasiliauskas, S. E. Plevy, C. J. Landers et al., "Perinuclear antineutrophil cytoplasmic antibodies in patients with Crohn's disease define a clinical subgroup," Gastroenterology, vol. 110, no. 6, pp. 1810-1819, 1996.

[14] N. Jamar-Leclerc, D. Reumaux, P. Duthilleul, and J. F. Colombel, "Do pANCA define a clinical subgroup in patients with Crohn's disease?" Gastroenterology, vol. 112, no. 1, pp. 316-317, 1997.

[15] L. Prideaux, M. Kamm A, P. de Cruz et al., "Inflammatory bowel disease serology in Asia and the West," World Journal of Gastroenterology, vol. 19, no. 37, pp. 6207-6213, 2013. 
[16] F. Rieder, R. Lopez, A. Franke et al., "Characterization of changes in Serum anti-glycan antibodies in Crohn's disease-a longitudinal analysis," PLoS ONE, vol. 6, no. 5, Article ID e18172, 2011.

[17] L. T. Vaszar, N. M. Orzechowski, U. Specks et al., "Coexistent pulmonary granulomatosis with polyangiitis (wegener granulomatosis) and Crohn disease," The American Journal of Surgical Pathology, vol. 38, no. 3, pp. 354-359, 2014.

[18] H. Yamashita, Y. Ueda, H. Kawaguchi et al., "Systemic lupus erythematosus complicated by Crohn's disease: a case report and literature review," BMC Gastroenterology, vol. 12, no. 1, article 174, 2012.

[19] A. Taddio, M. Maschio, S. Martelossi et al., "Crohns disease and Takayasus arteritis: an uncommon association," World Journal of Gastroenterology, vol. 19, no. 35, pp. 5933-5935, 2013. 


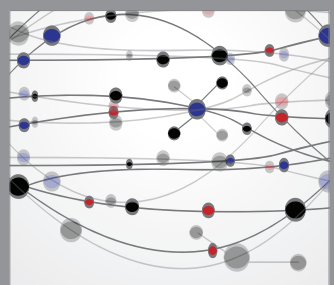

The Scientific World Journal
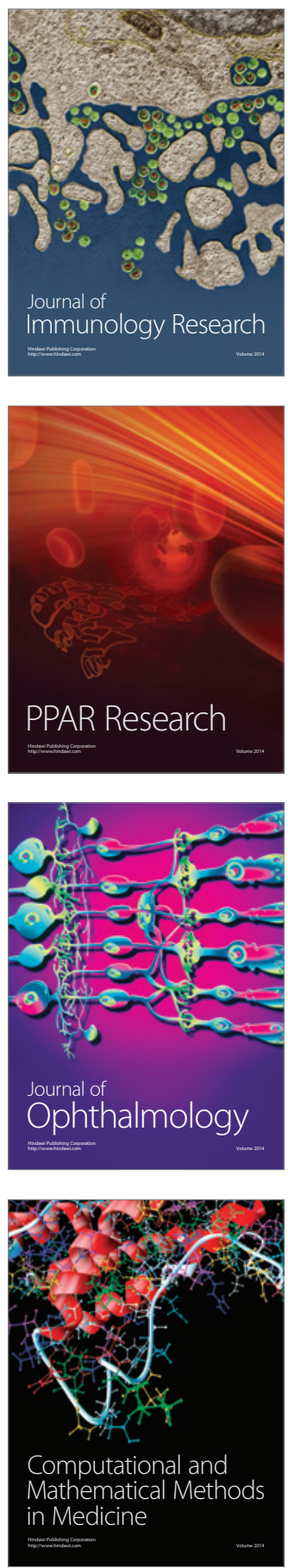

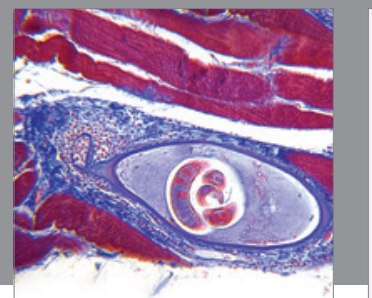

Gastroenterology

Research and Practice
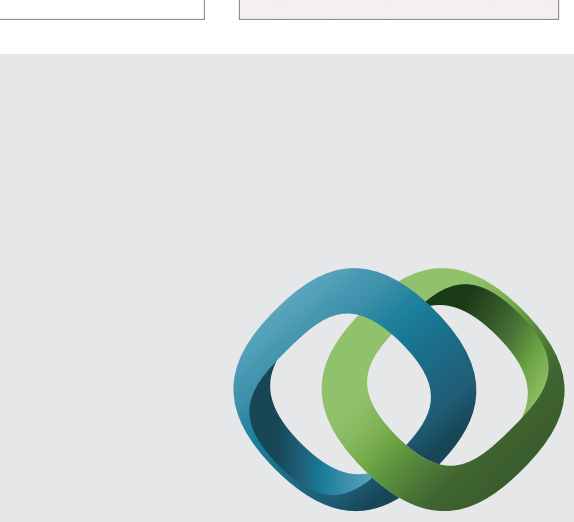

\section{Hindawi}

Submit your manuscripts at

http://www.hindawi.com
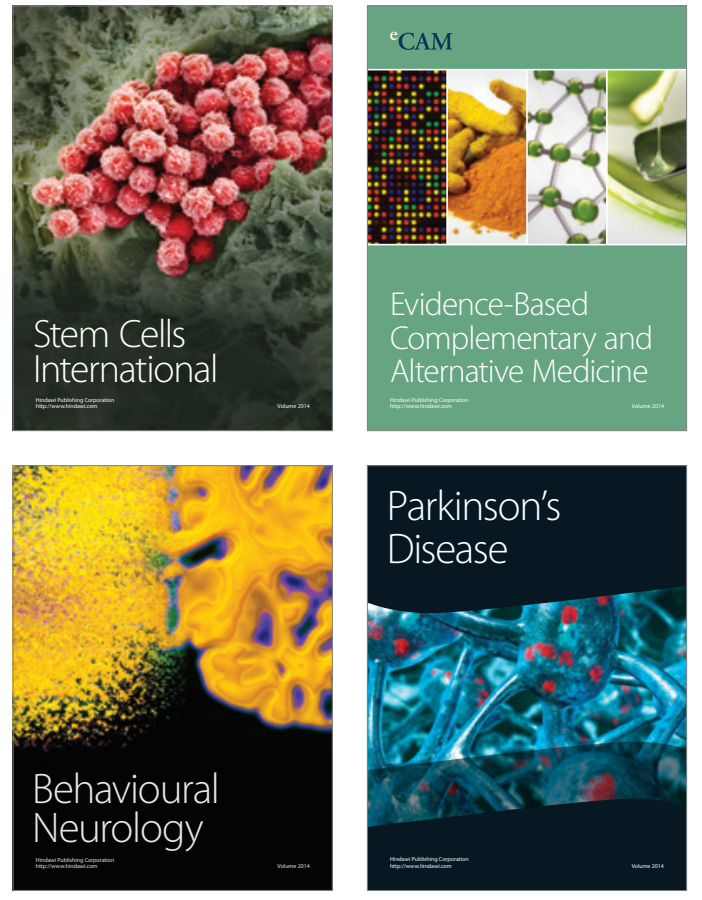
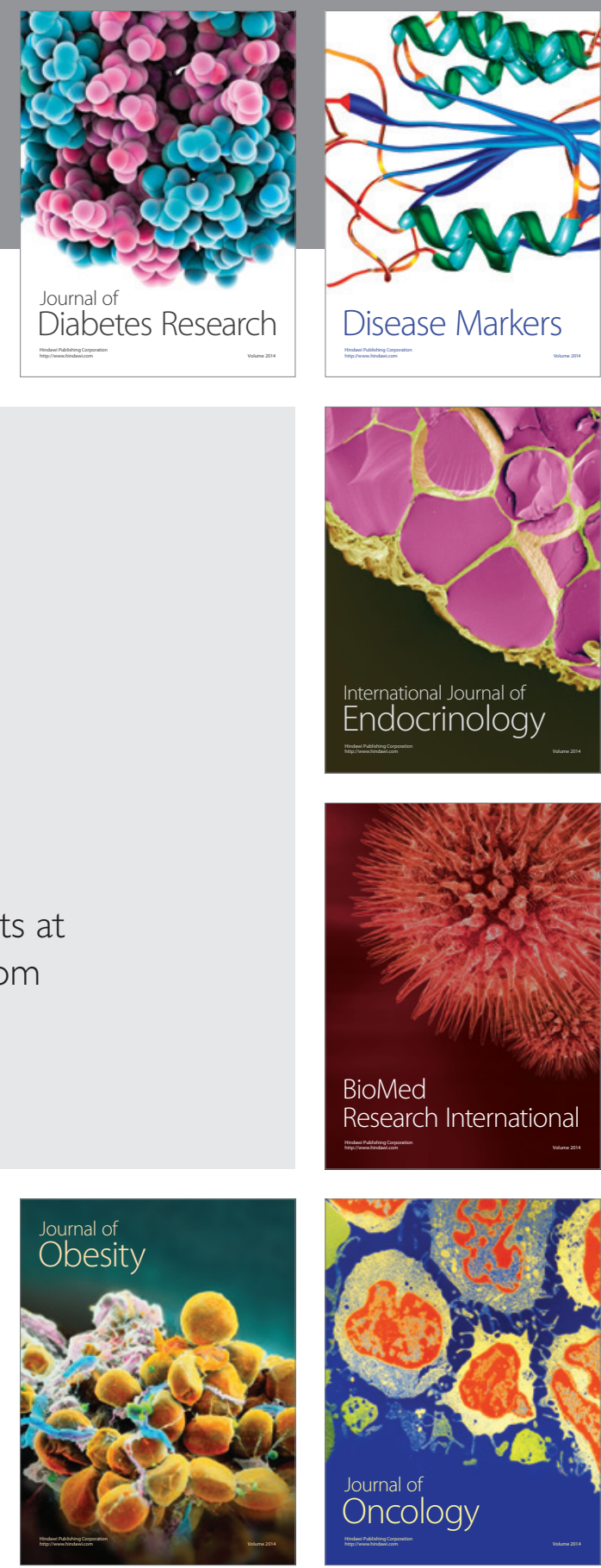

Disease Markers
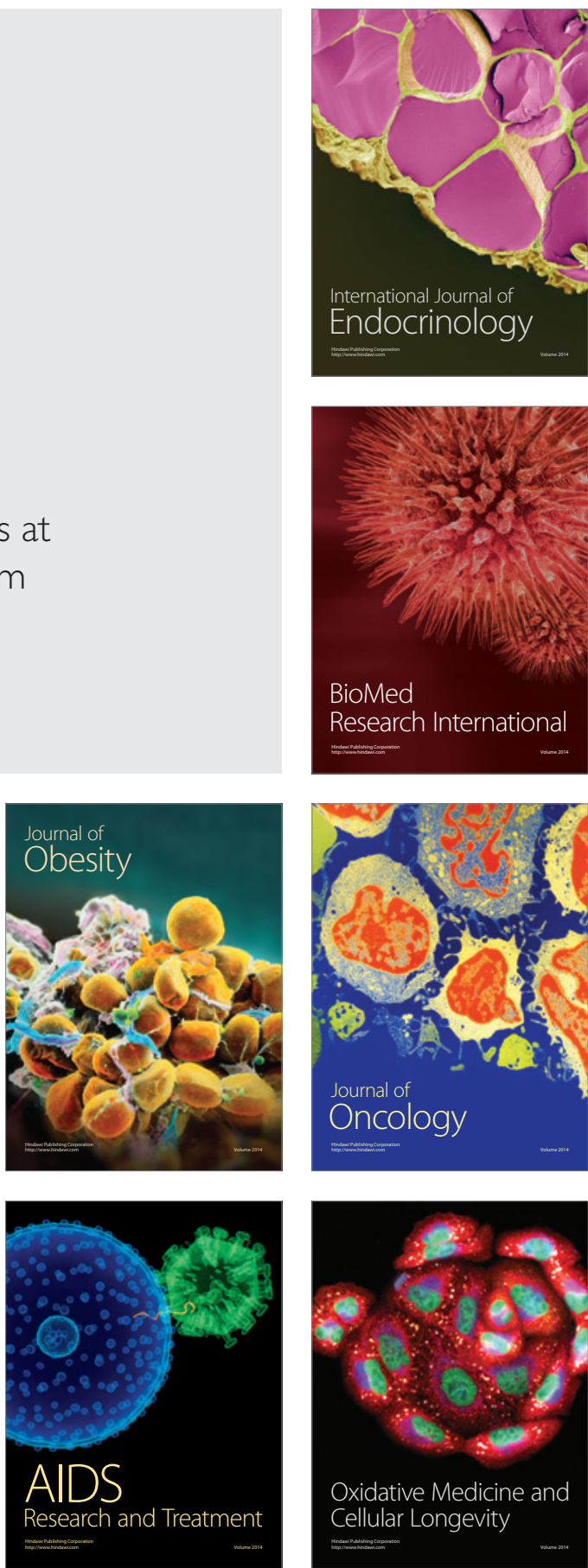